\title{
A Critical Role for the Anterior Thalamus in Directing Attention to Task-Relevant Stimuli
}

\author{
Nick F. Wright, Seralynne D. Vann, John P. Aggleton, and Andrew J.D. Nelson \\ School of Psychology, Cardiff University, Cardiff CF10 3AT, United Kingdom
}

\begin{abstract}
The prefrontal cortex mediates adaption to changing environmental contingencies. The anterior thalamic nuclei, which are closely interconnected with the prefrontal cortex, are important for rodent spatial memory, but their potential role in executive function has received scant attention. The current study examined whether the anterior thalamic nuclei are involved in attentional processes akin to those of prefrontal regions. Remarkably, the results repeatedly revealed attentional properties opposite to those of the prefrontal cortex. Two separate cohorts of rats with anterior thalamic lesions were tested on an attentional set-shifting paradigm that measures not only the ability of stimuli dimensions that reliably predict reinforcement to gain attention ("intradimensional shiff"), but also their ability to shift attention to another stimulus dimension when contingencies change ("extradimensional shift"). In stark contrast to the effects of prefrontal damage, anterior thalamic lesions impaired intradimensional shifts but facilitated extradimensional shifts. Anterior thalamic lesion animals were slower to acquire discriminations based on the currently relevant stimulus dimension but acquired discriminations involving previously irrelevant stimulus dimensions more rapidly than controls. Subsequent tests revealed that the critical determinant of whether anterior thalamic lesions facilitate extradimensional shifts is the degree to which the stimulus dimension has been established as an unreliable predictor of reinforcement over preceding trials. This pattern of performance reveals that the anterior thalamic nuclei are vital for attending to those stimuli that are the best predictors of reward. In their absence, unreliable predictors of reward usurp attentional control.
\end{abstract}

Key words: anterior thalamic nuclei; executive function; medial diencephalon; rat; set shifting

\section{Introduction}

The ability to adapt behavior in response to changing environmental contingencies is critical to the survival of an organism. Attentional set shifting is a form of behavioral flexibility that requires an organism to disengage from a previously relevant set of stimulus dimensions and, instead, respond to stimulus dimensions that have hitherto been experienced as irrelevant. This ability depends on the integrity of the dorsolateral prefrontal cortex in primates (Dias et al., 1996; Lombardi et al., 1999) and the medial prefrontal cortex in rodents (Birrell and Brown, 2000). Subcortical afferents to the prefrontal cortex are also known to contribute to behavioral flexibility (Hunt and Aggleton, 1998; Block et al., 2007; Lindgren et al., 2013). However, behavioral flexibility engages a range of different cognitive processes includ-

Received Dec. 3, 2014; revised Jan. 15, 2015; accepted Feb. 13, 2015.

Author contributions: S.D.V., J.P.A., and A.J.D.N. designed research; N.F.W. performed research; N.F.W. and A.J.D.N. analyzed data; S.D.V., J.P.A., and A.J.D.N. wrote the paper.

This work was support by the Wellcome Trust (Grants WT092480 and WT103723). S.D.V. is supported by a Wellcome Trust Senior Research Fellowship in Basic Biomedical Science (WT090954AIA). We thank Professor Steve Dunnett for the use of behavioral testing equipment, Dr. Emma Hindley for assistance with the behavioral task, and Professor John M. Pearce for discussion of theoretical models.

The authors declare no competing financial interests.

This article is freely available online through the J Neurosci Author Open Choice option.

Correspondence should be addressed to Andrew J. D. Nelson, School of Psychology, Cardiff University, Tower Building, Park Place, Cardiff CF10 3AT, U.K. E-mail: NelsonA5@cardiff.ac.uk.

DOI:10.1523/JNEUROSCI.4945-14.2015

Copyright $\odot 2015$ Wright et al.

This is an Open Access article distributed under the terms of the Creative Commons Attribution License Creative Commons Attribution 4.0 International, which permits unrestricted use, distribution and reproduction in any medium provided that the original work is properly attributed. ing attention to relevant stimuli, suppression of irrelevant information, and adaption to changing contingencies (Pearce and Mackintosh, 2010). These processes may depend on distinct neural pathways.

The anterior thalamic nuclei (ATN) are densely connected with the prefrontal cortex (Shibata, 1993; Shibata and Naito, 2005; Wright et al., 2013), though the functional significance of these interconnections is poorly understood. Executive dysfunction has been reported in patients with anterior thalamic damage (Ghika-Schmid and Bogousslavsky, 2000; Van der Werf et al., 2003; Lanna et al., 2012), and the anterior thalamic radiation, consisting of fibers connecting the thalamus with the prefrontal cortex, has also been implicated in higher-order cognition (Mamah et al., 2010; Duering et al., 2011; Biesbroek et al., 2013). However, as diverse areas within the thalamus may contribute to these effects, coupled with the lack of patients with circumscribed pathology, the neuroanatomical locus of these effects is debatable (Mitchell et al., 2014). This consideration highlights the particular merit of comparative lesion studies of this region.

For these reasons, rats with anterior thalamic nuclei lesions were tested on an attentional set-shifting task that measures attention to both relevant and irrelevant environmental stimuli, as well as the ability to adapt behavior in response to changing contingencies (Birrell and Brown, 2000; Fox et al., 2003; Ng et al., 2007; Chase et al., 2012). Rats receive a series of two-choice discriminations based on either odors or digging media, which form two perceptual dimensions. Rats undergo a series of four intradimensional (ID) shifts, during which one of the perceptual dimen- 
sions (e.g., odor) is consistently rewarded, while the other dimension (e.g., digging media) is irrelevant (Chase et al., 2012; Lindgren et al., 2013). Subsequently, the rats experience an extradimensional (ED) shift in which the previously irrelevant dimension (e.g., digging media) is now rewarded, thereby requiring a shift in attention away from the previously relevant dimension (e.g., odor). We tested two separate cohorts of rats, both of which received cytotoxic lesions in the anterior thalamic nuclei before training on this task. To test the generality of the observed effects, the second cohort was also challenged with additional extradimensional shifts involving the spatial properties of the testing environment, as well as a novel dimension that had not previously been experienced as irrelevant over successive preceding trials.

\section{Materials and Methods}

The set-shifting task used in the current study involves $3 \mathrm{~d}$ of pretraining as well as a further day of testing during which each animal acquires eight consecutive discriminations in a single session and the duration of this session is determined by the animal's own behavior (see below). As such, this experimental design does not lend itself to manipulations that temporarily silence a neural structure. Given these considerations, we targeted the anterior thalamic nuclei using cytotoxic lesions, an approach that provides the appropriate temporal resolution for this behavioral paradigm.

Subjects. The first experiment involved 25 adult male Lister Hooded rats (Charles River Laboratories). The rats weighed 270-320 g at the beginning of the experiment and were housed in pairs under a $12 \mathrm{~h}$ light/dark cycle. The rats either sustained bilateral lesions of the ATN $($ ATNx1 $=10)$ or sham surgeries $($ Sham $1=15)$. All animals were habituated to handling before the start of the first experiment.

Experiment 2 involved a cohort of 30 male Lister Hooded rats (Charles River Laboratories). The rats weighed $270-288 \mathrm{~g}$ at the beginning of the experiment. Housing and maintenance conditions matched those for Experiment 1 . The rats either received bilateral lesions of the ATN $($ ATNx2 $=16)$ or sham surgeries (Sham2 $=14)$.

All experiments were performed in accordance with the UK Animals (Scientific Procedures) Act (1986) and associated guidelines, as well as European Union directive 2010/63/EU. The study was also approved by local ethical review committees at Cardiff University.

Surgery. In Experiment 1, for 21 of the 25 rats the surgery was performed under an isoflurane-oxygen mixture (1.5-2.5\% isoflurane) with a reduced dose of sodium pentobarbital ( $14 \mathrm{mg} / \mathrm{kg}$, i.p.) when the surgery was nearing completion. For four rats, the surgeries involved just sodium pentobarbital anesthesia (60 mg/kg, i.p.; Sigma-Aldrich). Once anesthetized, the animal was placed in the head-holder of the stereotaxic apparatus (Kopf Instruments) with the incisor bar adjusted to +5.0 relative to the horizontal plane. Following an incision, the scalp was retracted to expose the skull. A craniotomy was made, and the dura was cut, exposing the cortex above the target location. Lesions in the anterior thalamic nuclei were made by injecting 0.12 м NMDA (Sigma Chemicals) dissolved in sterile phosphate buffer, $\mathrm{pH}$ 7.4, into two separate sites within each hemisphere using a $1 \mu \mathrm{l}$ Hamilton syringe. The syringe was attached to a moveable arm mounted on the stereotaxic frame. The injection site coordinates were as follows: medial injections: anteroposterior (AP), -0.1 ; mediolateral (ML), \pm 0.8 ; dorsoventral (DV), -6.8 ; lateral injections: $\mathrm{AP},-0.4 ; \mathrm{ML}, \pm 1.5 ; \mathrm{DV},-6.2$. Each of the medial injections consisted of $0.20 \mu \mathrm{l}$ of $0.12 \mathrm{M}$ NMDA, while the more lateral injections consisted of $0.18 \mu \mathrm{l}$ of $0.12 \mathrm{M}$ NMDA. Each injection was infused over a period of $5 \mathrm{~min}$. The syringe was left in situ for an additional $4 \mathrm{~min}$ before being retracted. For the sham surgeries, the syringe was lowered to +0.2 above the target site for a few seconds, and then removed. No NMDA was injected into these rats.

After removal of the Hamilton syringe, the incision was cleaned and sutured. A topical antibiotic powder (Aureomycin, Fort Dodge Animal Health) was applied. The rats received glucose-saline $(5 \mathrm{ml}$, s.c.) for fluid replacement and were then placed in a recovery chamber until they regained consciousness. Rats were given the analgesic metacam $(0.06 \mathrm{ml}$,

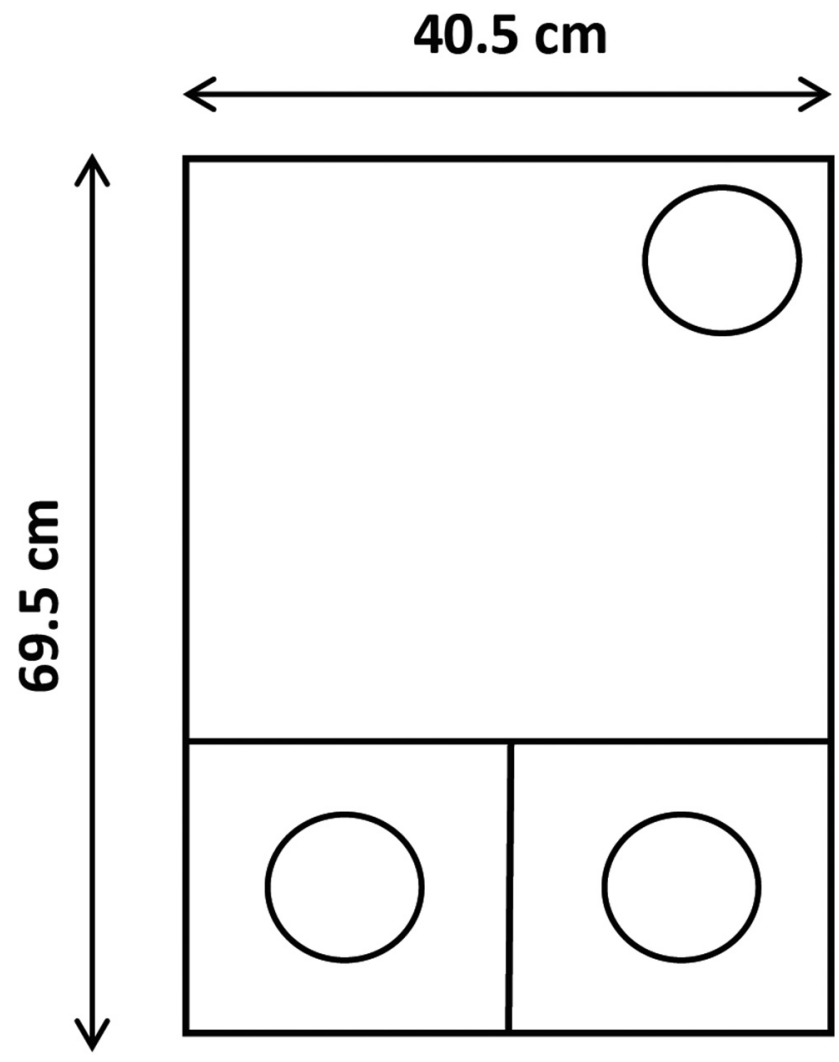

Figure 1. A schematic diagram of the apparatus used to run the set-shifting experiments. Approximately one third of the length of the box was divided into two smaller compartments, with the remaining area of the box being a single open space. The two smaller sections could be separated from the larger area by a removable black Perspex panel to control access. A glass pot (the clear circles), which was placed in each of the two smaller areas, contained digging medium. A third identical glass pot containing water was placed against the opposite wall of the larger area.

s.c.; $5 \mathrm{mg} / \mathrm{ml}$ meloxicam; Boehringer Ingelheim Vetmedica). A respiratory stimulant, Millophyline ( $0.1 \mathrm{ml}$, s.c.; Arnolds Veterinary Products), an antibiotic (Baytril 2.5\%; Bayer Ltd, Animal Health Division) in their water, and a low dose of diazepam $(0.07 \mathrm{ml}$, s.c.; $5 \mathrm{mg} / \mathrm{ml}$; CP Pharmaceuticals Ltd) were administered to facilitate postoperative recovery. All animals were monitored carefully until they had fully recovered.

In Experiment 2, the surgery was performed under an isofluraneoxygen mixture (1.5-2.5\% isoflurane), with a reduced dose of sodium pentobarbital ( $14 \mathrm{mg} / \mathrm{kg}$, i.p) when the surgery was nearing completion to prevent postoperative seizures. Experiment 2 used an equal mixture of ibotenic acid (10 mg/ml; Sigma-Aldrich) and NMDA (10 mg/ml, SigmaAldrich) dissolved in $1 \mathrm{ml}$ of sterile $0.1 \mathrm{M}$ PBS. All other aspects of the surgery were the same as in Experiment 1.

Apparatus. Testing was performed in a black Perspex box measuring $69.5 \mathrm{~cm}$ long $\times 40.5 \mathrm{~cm}$ wide $\times 18.6 \mathrm{~cm}$ deep (Fig. 1). Approximately one third of the length of the box was divided into two smaller compartments, with the remaining area of the box being a single open space. The two smaller sections could be separated from the larger area by removable black Perspex panels that could be used by the experimenter to control access. Each of the three sections had a separately hinged transparent Perspex lid. One glass pot ( $75 \mathrm{~mm}$ in diameter, $45 \mathrm{~mm}$ deep), which was used to contain digging media, was placed in each of the two smaller areas. A third identical glass pot containing water was placed against the opposite wall of the larger area.

Behavioral training: attentional set-shifting task (experiments 1 and 2). After a minimum of $14 \mathrm{~d}$ of postoperative recovery, rats were gradually reduced to $85 \%$ of their free-feeding weights and maintained on a restricted diet throughout behavioral testing. In experiment 1 , the rats were first tested on a rodent analog of the Stroop task (Nelson et al., 2014) and 
then tested on the attentional set-shifting task 3 months postoperatively. The Stroop test involved instrumental training in standard conditioning chambers with light and noise stimuli and different food rewards than those used in the set-shifting task. This training is, therefore, unlikely to confound learning of the set-shifting task. Moreover in experiment 2, testing on the attentional set-shifting task commenced immediately after the postoperative recovery period.

During testing, rats were presented with a series of two-choice discriminations. The stimuli were multidimensional, with only one stimulus dimension (odor or texture) signaling reward. Each cohort of rats was run through the same initial experimental procedure, with the second cohort receiving an additional set of extradimensional shifts at the end of the procedure.

Pretraining began $3 \mathrm{~d}$ before testing and consisted of the following three stages: habituation to the arena; digging training in the arena; and pre-exposure to each of the odors and media in the arena. On the first day of pretraining, each rat was placed in the arena for $10 \mathrm{~min}$ to habituate to the apparatus. For the first pretraining day, both removable panels were taken out of the arena, so animals had access to all three chambers, but the glass pots were not present. On the second day of pretraining, rats were returned to the arena with both smaller chambers closed off and all three of the glass pots in place. The two glass pots in the smaller chambers were filled with bedding sawdust and baited with half a Cheerio (Nestle). The rats were then trained to dig in the pots to obtain the food reward. Initially, the food reward was placed on top of the sawdust but was progressively buried until the rats readily dug in the sawdust for the food reward.

Once the rats could reliably find the food reward, they were preexposed to the stimuli that they would subsequently encounter in the test (Table 1). The purpose of the pre-exposure stage was to prevent refusals in the subsequent test without the rats acquiring any stimulus-reward associations. Each odor that would be used was presented mixed with bedding sawdust. Digging media were presented without odors added. On the third day of pretraining, each rat was required to retrieve a buried Cheerio from each pot of digging medium and from each pot of odorlaced sawdust. This procedure was repeated in each of the smaller chambers, to avoid any spatial bias.

On the test day, rats were trained on a sequential series of discriminations (Table 1). The rat did not start the next discrimination until it had acquired the present discrimination, with all tests completed in a single day for each rat. The first discrimination was either between two different odors or between two different digging media (the choice of stimuli was counterbalanced between groups). For these discriminations, the glass pots in each of the smaller areas were filled with either two different digging media or sawdust infused with two different scents. Only one odor (or one medium) contained a buried Cheerio. The location of the rewarded bowl was pseudorandomly allocated in each trial. Once the dividing doors were removed, the rat had $10 \mathrm{~min}$ to find the Cheerio. If the rat dug in the rewarded pot-defined as breaking the surface of the digging medium with the paws or nose- the trial was marked as correct. If the rat dug in the incorrect pot, the trial was marked as incorrect. For the first four trials of each discrimination, the rat was allowed access to the correct pot to uncover the reward following an incorrect initial dig. On subsequent incorrect trials, the trial was terminated as soon as the rat had made a response and was not allowed access to the correct pot if it had made an incorrect response. The intertrial interval was $\sim 5 \mathrm{~s}$ long, during which time the cups were rebaited. The performance criterion, to move on to the next discrimination, was six correct choices in a row. An experimenter, blind to whether the rat was in the ATNx or Sham group, scored the number of trials to criterion as well as the total numbers of errors made in each trial.

During the simple discrimination (SD), rats dug in scented sawdust or in a digging medium with no odor added. In the subsequent compound discrimination $(\mathrm{CD})$, the same odor or digging medium rewarded in the simple discrimination was still rewarded, but a second irrelevant dimension was added. The next four stages of the task were ID acquisitions (ID1, ID2, ID3, and ID4), during which different compound stimuli were presented in each discrimination, with the relevant dimension remaining constant (Table 1). Thus, during the first six stages of the task
(SD, CD, ID1, ID2, ID3, and ID4) the rats were required to find rewards by attending to only one stimulus dimension (either odor or digging media, counterbalanced across groups) while ignoring the other nonrewarded dimension. This training should encourage the formation of an attentional set (i.e., always attend to one dimension and ignore the other, irrelevant, dimension). Improvements in performance across these six trials provide an index of successful set formation.

Thereafter, the rats were challenged with an ED shift. Different compound stimuli were again presented, but now the previously irrelevant dimension was rewarded. Thus, the rats were required to refocus their attention to the previously irrelevant dimension to solve the discrimination. Typically rats show a "shift cost" as more trials are required to learn the ED discrimination relative to the preceding ID discrimination (Birrell and Brown, 2000). This shift cost provides an index of the ability to shift attention to previously irrelevant stimuli. Thereafter, the rats underwent a reversal (REV) in which the reward contingencies were reversed (Table 1).

Additional spatial and novel extradimensional shifts (experiment 2 only). In two separate cohorts and under identical experimental conditions, ATN lesions appeared to facilitate the acquisition of an ED shift. We, therefore, sought to establish whether this apparent advantage could be reproduced under different experimental conditions (i.e., an ED shift that did not involve a switch between odor and digging material, or vice versa). Throughout the $3 \mathrm{~d}$ of pretraining and the eight discriminations comprising the set-shifting task, the spatial location of the rewarded pot (in either the left or the right chamber of the testing box) was pseudorandomly assigned such that its location was irrelevant to solving the discrimination. After the completion of initial training, the rats in experiment 2 were, therefore, exposed to an additional ED shift based on the spatial location of the rewarded pot (Table 1). In addition, we challenged the rats with a further ED shift that required the animals to learn about a relatively novel dimension that had not been experienced as irrelevant throughout all preceding testing (Table 1). To this end, we introduced a novel dimension (Velcro encasing) to the pots. As the attention paid to novel stimuli is assumed to be high, discriminations involving novel stimuli may be acquired more readily (Pearce and Hall, 1980). So that the dimension was not entirely novel, it was introduced at the beginning of the additional training stage. For the same reason, all animals underwent the series of additional discriminations in the same order (Table 1). If anterior thalamic lesions generally facilitate attentional set shifting, then the ATNx group should acquire the discrimination involving the novel dimension more rapidly than sham animals. If, however, the critical determinant of whether anterior thalamic lesions facilitate ED shifts is the extent to which the now relevant domain has previously been experienced as irrelevant, then the ATNx group should learn about the novel dimension at the same rate as the control animals.

The rats underwent this subsequent training in the same testing box but with adapted pots. Velcro was placed around the edge of the pots such that half the pots were encased with a strip of Velcro Hook and the other half with Velcro Loop. First, the rats acquired a compound discrimination followed by an ED shift, its reversal, and, finally, a second ED shift (Table 1). For the compound discrimination, the rats were required to discriminate the pots on the basis of the digging medium and ignore the Velcro/spatial location. For the spatial ED shift, the reward was now placed in a digging pot in a specific spatial location (either the left-hand or right-hand chamber of the testing box) regardless of either the digging medium or the Velcro. To solve this spatial ED shift, the rats were required to ignore both the digging media and the Velcro, and to attend to the previously irrelevant spatial location of the pot. Once this ED discrimination was acquired, the contingencies were reversed such that the previously unrewarded chamber was now rewarded (e.g., reversal from left chamber to right chamber). Thereafter, the rats underwent a further ED shift in which the Velcro encasing (either hook or loop), but not the digging medium or spatial location, determined which pot was rewarded (Table 1).

Histology. Following behavioral testing, the animals were administered an intraperitoneal injection of a lethal overdose of Euthatal $(200 \mathrm{mg} / \mathrm{ml}$ sodium pentobarbital; Marial Animal Health Ltd.) and perfused intracardially with $0.1 \mathrm{M}$ PBS followed by $4 \%$ paraformaldehyde (PFA) in $0.1 \mathrm{M}$ 
Table 1. Depiction of a possible set of stimulus pairings for the attentional set-shifting task

\begin{tabular}{|c|c|c|}
\hline \multirow[b]{2}{*}{ Discrimination } & \multicolumn{2}{|l|}{ Stimuli } \\
\hline & Rewarded & Unrewarded \\
\hline \multicolumn{3}{|c|}{ Experiments 1 and $2^{*}$} \\
\hline SD odor & Oregano & Cloves \\
\hline & Oregano + shredded paper & Cloves + confetti \\
\hline ID1 odor & $\begin{array}{l}\text { Cinnamon }+ \text { coarse tea } \\
\text { or } \\
\text { Cinnamon }+ \text { fine tea }\end{array}$ & $\begin{array}{l}\text { Ginger }+ \text { fine tea } \\
\text { or } \\
\text { Ginger }+ \text { coarse tea }\end{array}$ \\
\hline ID2 odor & $\begin{array}{l}\text { Tarragon + coarse cork } \\
\text { or } \\
\text { Tarragon + fine cork }\end{array}$ & $\begin{array}{l}\text { Fenugreek + fine cork } \\
\text { or } \\
\text { Fenugreek + coarse cork }\end{array}$ \\
\hline ID3 odor & $\begin{array}{l}\text { Marjoram + wood shavings } \\
\text { or } \\
\text { Marjoram + wood chip }\end{array}$ & $\begin{array}{l}\text { Sage }+ \text { wood chip } \\
\text { or } \\
\text { Sage }+ \text { wood shaving }\end{array}$ \\
\hline ID4 odor & $\begin{array}{l}\text { Cumin }+ \text { short cigarette filters } \\
\text { or } \\
\text { Cumin }+ \text { long cigarette filters }\end{array}$ & $\begin{array}{l}\text { Dill + long cigarette filters } \\
\text { or } \\
\text { Dill + short cigarette filters }\end{array}$ \\
\hline ED medium & $\begin{array}{l}\text { Polystyrene }+ \text { mint } \\
\text { or } \\
\text { Polystyrene }+ \text { turmeric }\end{array}$ & $\begin{array}{l}\text { Beanbag filler + turmeric } \\
\text { or } \\
\text { Beanbag filler }+ \text { mint }\end{array}$ \\
\hline REV medium & $\begin{array}{l}\text { Beanbag Filler + turmeric } \\
\text { or } \\
\text { Beanbag Filler }+ \text { mint }\end{array}$ & $\begin{array}{l}\text { Polystyrene }+ \text { mint } \\
\text { or } \\
\text { Polystyrene + turmeric }\end{array}$ \\
\hline \multicolumn{3}{|c|}{ Experiment 2 only $\dagger$} \\
\hline CD medium & $\begin{array}{l}\text { Short wire + Velcro loop } \\
\text { or } \\
\text { Short wire + Velcro hook }\end{array}$ & $\begin{array}{l}\text { Long wire + Velcro hook } \\
\text { or } \\
\text { Long wire + Velcro loop }\end{array}$ \\
\hline EDspatial & $\begin{array}{l}\text { Left chamber + short wire + Velcro loop } \\
\text { or } \\
\text { Left chamber + short wire + Velcro hook } \\
\text { or } \\
\text { Left chamber + long wire + Velcro hook } \\
\text { or } \\
\text { Left chamber + long wire + Velcro loop }\end{array}$ & $\begin{array}{l}\text { Right chamber + short wire + Velcro loop } \\
\text { or } \\
\text { Right chamber + short wire + Velcro hook } \\
\text { or } \\
\text { Right chamber + long wire + Velcro hook } \\
\text { or } \\
\text { Right chamber + long wire + Velcro loop }\end{array}$ \\
\hline REVspatial & $\begin{array}{l}\text { Right chamber + short wire + Velcro loop } \\
\text { or } \\
\text { Right chamber + short wire + Velcro hook } \\
\text { or } \\
\text { Right chamber + long wire + Velcro hook } \\
\text { or } \\
\text { Right chamber + long wire + Velcro loop }\end{array}$ & $\begin{array}{l}\text { Left chamber + short wire + Velcro loop } \\
\text { or } \\
\text { Left chamber + short wire + Velcro hook } \\
\text { or } \\
\text { Left chamber + long wire + Velcro hook } \\
\text { or } \\
\text { Left chamber + long wire + Velcro loop }\end{array}$ \\
\hline EDnovel & $\begin{array}{l}\text { Velcro loop + right chamber + short wire } \\
\text { or } \\
\text { Velcro loop + right chamber + long wire } \\
\text { or } \\
\text { Velcro loop + left chamber + short wire } \\
\text { or } \\
\text { Velcro loop + left chamber + long wire }\end{array}$ & $\begin{array}{l}\text { Velcro hook + right chamber + short wire } \\
\text { or } \\
\text { Velcro hook + right chamber + long wire } \\
\text { or } \\
\text { Velcro hook + left chamber + short wire } \\
\text { or } \\
\text { Velcro hook + left chamber + long wire }\end{array}$ \\
\hline
\end{tabular}

Rewarded domain is in bold.

*Depiction of a possible set of stimulus pairings for the attentional set-shifting task. A food reward is buried in the correct pot and rats dig to find it. The pots are discriminable by either the digging medium or the odor. In the example given, the odor discrimination is relevant until the ED shift stage, when the medium discrimination becomes relevant. After the SD, rats are presented with compound stimuli comprising one stimulus from the relevant domain and one stimulus from the irrelevant domain. The stimuli are always presented in these combinations, but the stage at which the combination is encountered is counterbalanced across animals.

tDepiction of a possible set of stimulus pairings for the additional discriminations (experiment 2 only). A food reward is buried in the correct pot and rats dig to find it. The pots are discriminable by digging media, encasing, or spatial location. After acquiring a discrimination based on digging media (CD), the spatial location of the pot becomes relevant (EDspatial shift), then the opposite spatial location becomes relevant (REVspatial ), and finally the encasing of the pot becomes relevant (EDnovel shift).

PBS. The brains were extracted from the skull and placed on a stirrer to postfix in PFA for $4 \mathrm{~h}$, after which the brains were placed in $25 \%$ sucrose overnight. The brains were frozen on a microtome (Leica) and sectioned at $40 \mu \mathrm{m}$ in the coronal plane. One in five sections was mounted and stained with cresyl violet, a Nissl stain.

Volumetric analysis. The extent of the lesions in the anterior thalamic nuclei was first drawn by hand onto five equidistant coronal sections (Paxinos and Watson, 2005). These images were scanned, and the area of damage was quantified using the program analySIS D (Soft Imaging System GmbH/Olympus). The percentage of damage to the anterior thalamic nuclei was quantified by taking the area of damage within the region of interest and dividing it by the total area of that region summed across each drawing.

Data analysis. Trials to the criterion for the attentional set-shifting task were analyzed by two-way ANOVA with "stage" (eight levels) as a repeated-measures factor, and "lesion" as a between-subjects factor. When significant interactions between stage and lesion were found, simple main-effects analyses were conducted by an additional ANOVA restricted to the relevant factors. The $F$ values associated with the simple main effects or simple interactions were recomputed by using the appropriate error term and degrees of freedom. All statistical analyses were computed using SPSS (version 20.0). The $\alpha$ level was set at $p<0.05$. 

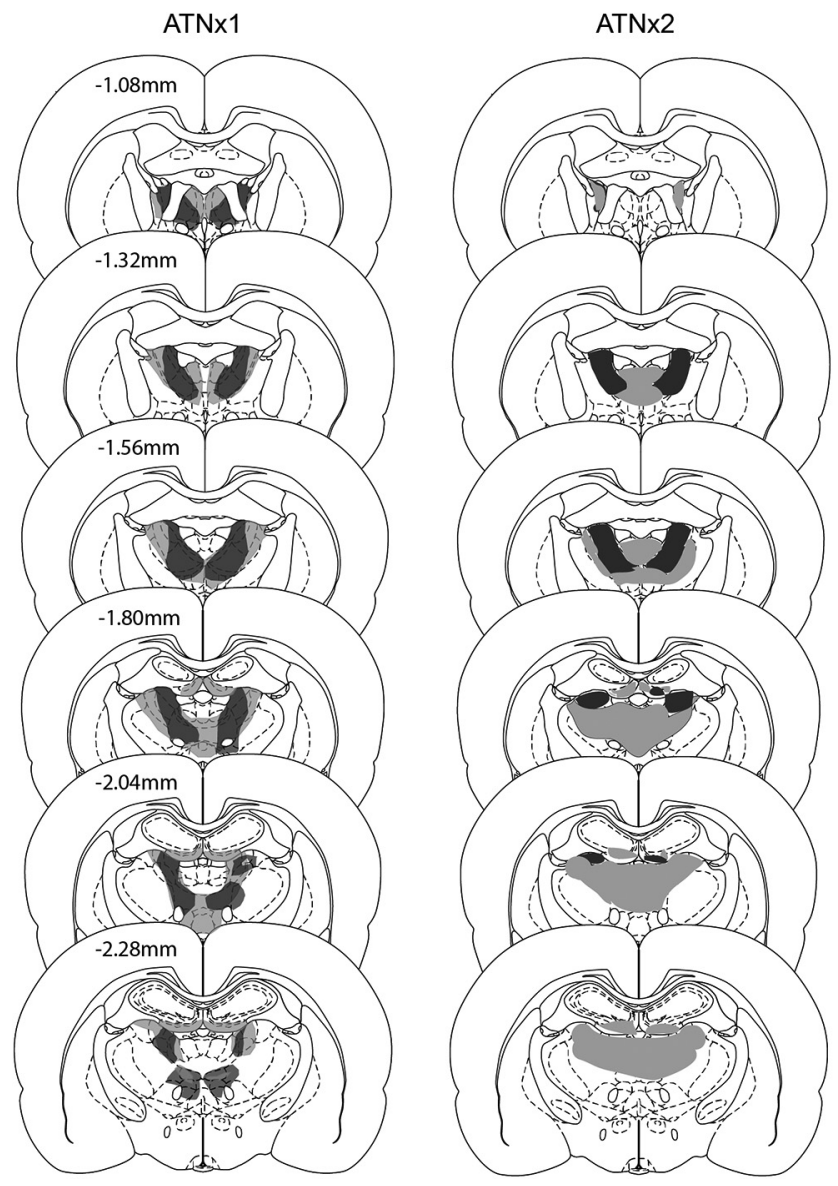

Figure 2. Depiction of the cases with the minimum (dark gray) and maximum (light gray) extent of cell loss in the ATNx1 and ATNx2 groups. The numbers refer to the approximate distance (in millimeters) of each section caudal to bregma. The coronal sections are adapted from Figures 42, 44, 46, 48, 50, and 52 in Paxinos and Watson, 2005, with permission.

\section{Results}

\section{Histology}

Experiment 1

Of the 10 rats, two had excessive, unintended cell loss within the medial dentate gyrus of the septal hippocampus and so were excluded from the study. All subsequent descriptions relate to the remaining eight $\mathrm{ATNx} 1$ rats (Fig. 2). In these eight cases, the anterior thalamic nuclei lesions were either essentially complete $(n=4)$ or a small island of cells within the anterior ventral nucleus was visible in just one hemisphere. Consequently, the range of tissue loss from the anterior thalamic nuclei was $82-98 \%$ ( mean $=92 \%$; median $=95 \%)$. Some of the lesions extended into adjacent midline nuclei such as the paraventricular nucleus $(n=4)$ and parataenial nucleus $(n=4,3$ of which had only unilateral cell loss). The lesions sometimes also extended ventrally to reach the very rostral part of the reticular nucleus and the ventral anterior nucleus (both, $n=3$ ). The rostral nucleus reuniens was involved in seven cases. More caudal nuclei such as the mediodorsal thalamic nucleus (unilateral, three cases) and the lateral dorsal nucleus (three cases, two of which were unilateral) were occasionally involved at their rostral limit. Cell loss within the hippocampus was seen in only three cases, where it was typically restricted to the ventral blade of the dentate gyrus in the most rostral part of the septal hippocampus. A more common feature was that the third and lateral ventricles appeared enlarged.

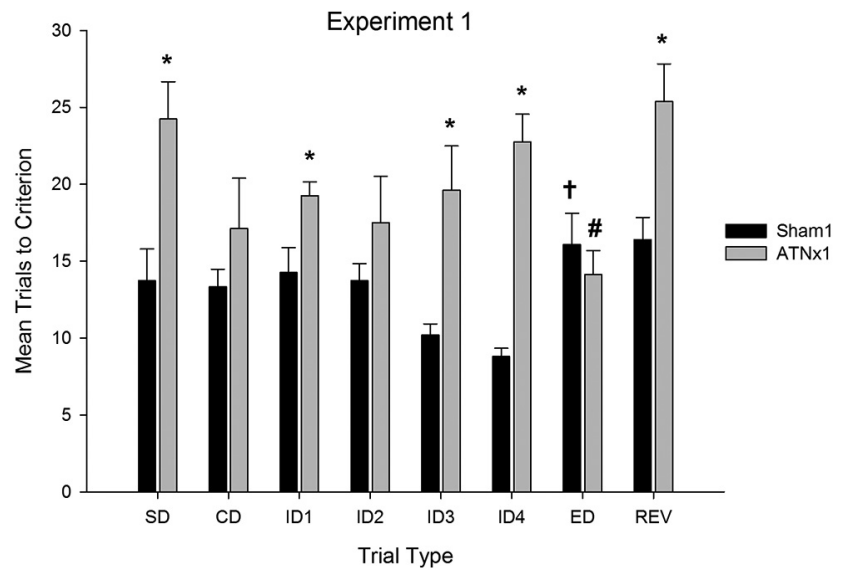

Figure 3. Mean (+SEM) trials to criterion data on the attentional set-shifting task (experiment 1). ATNx1 rats were significantly slower to learn several stages of the task ( ${ }^{*}$ significantly different from controls, $p<0.05$ ). Sham-treated rats required more trials to learn the ED shift stage than the fourth intradimensional shift (†significantly different from the last ID shift, $p<$ 0.05 ), but the ANTX1 rats required fewer trials to complete the ED shift (\#significantly different from the last ID shift, $p<0.05)$.

\section{Experiment 2}

Of the 16 rats receiving anterior thalamic surgeries, one had considerable sparing in one hemisphere and was, therefore, rejected from all analyses. The remaining 15 rats received extensive bilateral lesions involving all of the anterior thalamic nuclei (Fig. 2). In general, the lesions in this cohort were more restricted to the target nuclei than in the previous experiment. The tissue loss from the anterior thalamic nuclei ranged from 53\% to $99 \%$ ( mean $=75 \%$; median $=78 \%$ ). In half of the 15 cases, the lesions extended into the immediately adjacent parataenial nucleus (four bilateral, four unilateral). The dorsal part of rostral nucleus reuniens was also partially involved in eight cases (six bilateral). The lesions also extended caudally to involve the rostral part of the lateral dorsal nucleus (five bilateral, two unilateral) and occasionally reached the most rostral part of the mediodorsal nucleus (two bilateral, one unilateral). Cell loss within the hippocampus was more frequent than that seen experiment 1, but when it did occur it was highly restricted. Any hippocampal damage was typically confined to the ventral blade of the dentate gyrus in the most rostral part of the septal hippocampus (seven bilateral, four unilateral).

\section{Behavioral testing}

Attentional set-shifting task: experiment 1

Initial analysis revealed no effects of modality (i.e., whether the rats were required to attend to odor or digging media) on performance or any interactions involving this factor and the lesion group $\left(\right.$ maximum $F_{(5,95)}=1.1, p=0.37$ ). Consequently, the data were pooled across the two dimensions for all analyses.

As is clear from Figure 3, rats in the ATNx1 group were slower than controls to form an attentional set, requiring more trials to complete several stages of the task (stages SD to ID4). ANOVA revealed an interaction between stage and lesion $\left(F_{(5,105)}=3.0\right.$, $p<0.05)$ as well as an overall effect of lesion $\left(F_{(1,21)}=37.3, p<\right.$ 0.001). Simple-effects analysis confirmed that the ATNx1 rats required more trials to criterion relative to sham-treated rats on the SD, ID1, ID3, and ID4 stages of the task (minimum $F_{(1,21)}=$ 4.6, $p<0.05$ ) but did not differ from the sham-treated rats on the $\mathrm{CD}$ and ID2 stages (maximum $F_{(1,21)}=2.03, p=0.17$ ).

As expected, the sham group showed a significant increase in the number of trials to reach criterion when they had to shift their 


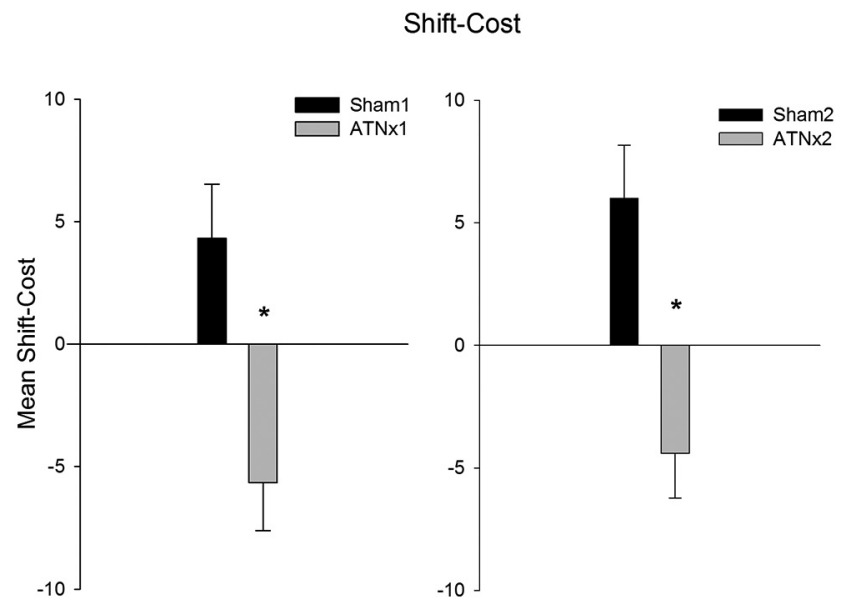

Figure 4. Mean ( \pm SEM) shift costs for each experiment. The shift cost is calculated by taking the difference in trials required to learn the extradimensional shift and the average number of trials across the four preceding intradimensional shifts. Sham-treated rats in both experiments show a positive shift cost requiring more trials to complete the extradimensional shift. Both cohorts of anterior thalamic lesion rats show a shift benefit (i.e., requiring fewer trials to complete the extradimensional shift; ${ }^{*}$ significantly different from controls, $p<0.05$ ).

attention to the previously irrelevant stimulus dimension (ED stage). In stark contrast, the ATNxl group acquired the ED shift more rapidly than the previous ID4 stage. ANOVA yielded no effect of stage, but did yield an interaction between stage (ID4 vs ED) and lesion $\left(F_{(1,21)}=20.4, p<0.001\right)$, as well as a significant effect of lesion $\left(F_{(1,21)}=13.64, p<0.001\right)$. This interaction arose because the sham-treated rats required more trials to complete the ED stage relative to ID4 $\left(F_{(1,21)}=8.53, p<0.01\right)$, whereas rats in the ATNx group required fewer trials $\left(F_{(1,21)}=12.01, p<\right.$ 0.001 ). Analysis of the shift cost (Fig. 4; i.e., comparing the mean number of trials to criterion across the four ID stages and the ED stage) confirmed a significant difference between the two groups $\left(F_{(1,21)}=8.8, p<0.01\right)$.

This advantage on the ED shift in the ATNx1 group did not reflect a nonspecific discrimination learning practice effect, because when the contingencies were reversed (stage REV) the ATNxl group took longer to learn the reversal than the previous ED shift (Fig. 3). An ANOVA, comparing performance on the ED and REV stages, revealed an effect of trial $\left(F_{(1,21)}=12.7, p<\right.$ $0.001)$ and also a trial X lesion interaction $\left(F_{(1,21)}=11.3, p<\right.$ $0.01)$, but no main effect of lesion $\left(F_{(1,21)}=2.3, p=0.15\right)$. This interaction arose because performance across the two stages was equivalent in the sham-treated group $(F<1)$, but the ATNx1 group required more trials to learn the reversal than the ED shift $\left(F_{(1,21)}=18.4, p<0.001\right)$.

Attentional set-shifting task: experiment 2

Whether the rats were initially trained to attend to odor or medium had no effect on any performance measure (maximum $\left.F_{(5,125)}=1.6, p=0.17\right)$, and so all of the data were analyzed by pooling them across the two dimensions.

The mean number of trials to criterion are displayed in Figure 5. As with experiment 1, the ATNx2 group was impaired relative to the control group on several stages of the attentional set formation task (stages SD to ID4). ANOVA confirmed a stage by lesion interaction $\left(F_{(5,135)}=3.6, p<0.005\right)$ as well as a main effect of lesion $\left(F_{(1,27)}=7.3, p<0.05\right)$, but no overall effect of stage $\left(F_{(5,135)}=1.4, p=0.24\right)$. Simple-effects analysis revealed that the rats in the ATNx2 group were slower to reach criterion on the SD and ID4 stages of the task (minimum $F_{(1,27)}=5.6, p<$

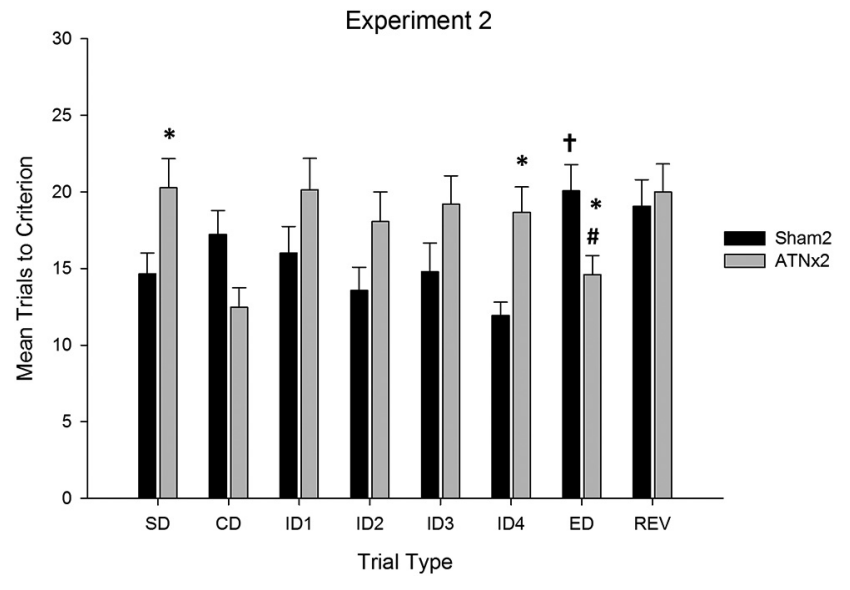

Figure 5. Mean ( + SEM) trials to criterion data on attentional set-shifting task (experiment 2). Rats with lesions in the anterior thalamus (ATNx2) were significantly slower to learn several stages of the task (*significantly different from controls, $p<0.05$ ). Sham-treated rats required more trials to learn the ED shift stage than the fourth intradimensional shift (†significantly different from the last ID shift, $p<0.05$ ), but the ANTx2 rats required fewer trials to complete the ED shift (\#significantly different from the last ID shift, $p<0.05$ ).

0.05), but did not differ statistically on the ID1, ID2, and ID3 stages (maximum $F_{(1,27)}=3.3, p=0.08$ ). In contrast, they were faster to reach criterion on the CD stage $\left(F_{(1,27)}=5.5, p<0.05\right)$.

The sham animals demonstrated a significant increase in the number of trials to criterion between the ID4 and ED stages, confirming a shift cost when required to learn about the previously irrelevant dimension. Conversely, the ATNx2 group showed a shift benefit, because they required fewer trials to complete the ED stage than the preceding ID4 stage. This description of the data was confirmed by ANOVA, which revealed no effect of stage $\left(F_{(1,27)}=2.2, p=0.16\right)$ or lesion $(F<1)$, but a significant lesion $\times$ stage interaction $\left(F_{(1,27)}=19.1, p<0.001\right)$. This interaction arose because the sham group required significantly more trials $\left(F_{(1,27)}=12.4, p<0.01\right)$, while the ATNx2 group required significantly fewer trials $\left(F_{(1,27)}=6.8, p<0.05\right)$ to complete the ED shift relative to the preceding ID4 stage. Analysis of the shift cost (Fig. 4) confirmed that the Sham 2 and ATNx2 groups differed significantly on this measure of performance $\left(F_{(1,27)}=13.7\right.$, $p<0.001)$.

When the reward contingencies were reversed (stage REV), the ATNx2 group took longer to learn the reversal than the preceding ED stage, but performance in the sham group was equivalent across the two stages. ANOVA yielded no effect of stage $\left(F_{(1,27)}=2.2, p=0.15\right)$ or lesion $\left(F_{(1,27)}=1.66, p=0.21\right)$ but a stage $\times$ lesion interaction $\left(F_{(1,27)}=4.6, p<0.05\right)$. Simple-effects analysis confirmed that rats in the ATNx2 group $\left(F_{(1,27)}=6.7\right.$, $p<0.05)$, but not the sham-treated rats $(F<1)$, demonstrated an increase in the number of trials to complete when the contingencies were reversed.

The number of rats without any damage to nucleus reuniens, which is also interconnected with prefrontal cortex, made it possible to compare performance in the two subgroups of rats with or without damage to this thalamic nucleus. Focusing on the key shift-cost measure of extradimensional shift performance, there was no evidence that nucleus reuniens damage contributed to the pattern of results obtained $(F<1)$.

Additional extradimensional shifts: experiment 2

The performance on the additional stages, in which the animals experienced an ED shift based on the spatial location of the pot 


\section{Experiment 2: Additional Extra-dimensional Shifts}

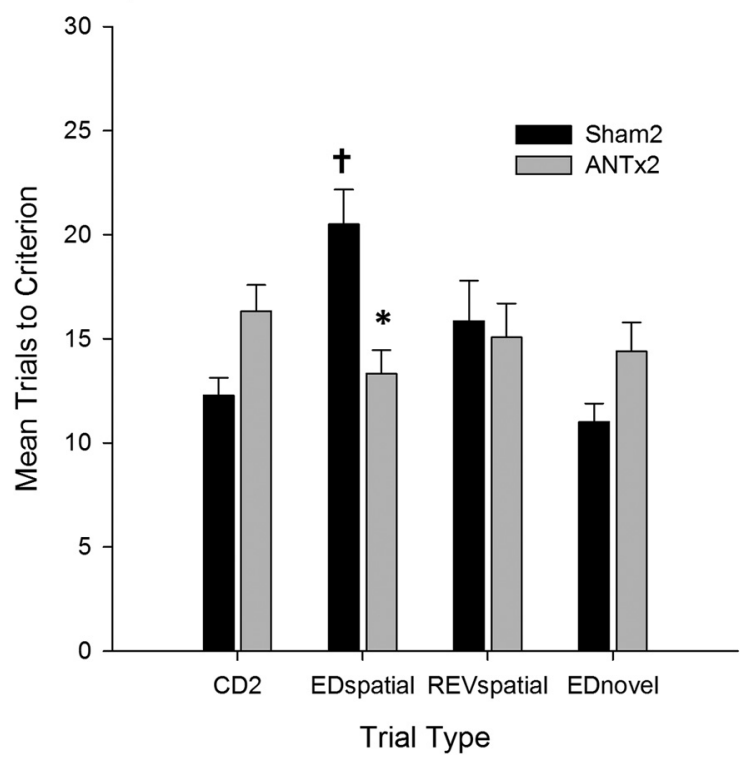

Figure 6. Mean (+SEM) trials to criterion data on the additional extradimensional shifts (experiment 2). ATNx2 rats were significantly faster to learn the spatial extradimensional shift ( ${ }^{*}$ significantly different from controls, $p<0.05$ ). Sham-treated rats required more trials to learn the ED shift stage than the previous discrimination (†significantly different from previous discrimination, $p<0.05$ ). REVspatial, REV involving spatial location of the pot.

(Fig. 6), as well as an ED shift based on a novel dimension (material encasing the pot), differed by lesion group. ANOVA confirmed an effect of stage $\left(F_{(3,81)}=3.3, p<0.05\right)$, a stage $\times$ lesion interaction $\left(F_{(3,81)}=6.9, p<0.001\right)$, but no overall effect of lesion. Simple effects confirmed that the ATNx2 group took longer to complete the compound discrimination $\left(F_{(1,27)}=7.1 p<\right.$ $0.05)$, marginally longer to complete the ED shift involving a novel dimension (i.e., pot encasing; EDnovel; $F_{(1,27)}=4.1, p=$ $0.052)$, did not differ on the spatial reversal $(F<1)$ and were significantly quicker to complete the ED shift involving the spatial location of the pot (EDspatial; $F_{(1,27)}=13.0, p<0.001$ ). Further simple-effects analysis revealed a significant shift cost on the EDspatial shift $\left(F_{(1,27)}=19.4, p<0.001\right)$ in the sham-treated group, because these animals took longer to acquire the EDspatial shift relative to the previous compound discrimination (Fig. 6). This shift cost was absent in the ATNx2 group, and in fact there was a trend toward a shift benefit, albeit one that narrowly failed to reach statistical significance $\left(F_{(1,27)}=4.1, p=0.06\right)$. For the final ED shift, the animals were required to learn a discrimination based on a novel dimension. There was no evidence that either group took longer to acquire the novel ED shift, and, if anything, there was a trend toward acquiring the novel discrimination more rapidly than the previous REV stage (maximum $F_{(1,27)}=2.9, p=$ $0.1)$. This trend did not interact with lesion $\left(F_{(1,27)}=1.7, p=0.21\right)$.

\section{Discussion}

The current experiments examined how lesions centered in the anterior thalamic nuclei affect a set-shifting task that measures both intradimensional and extradimensional attention shifts. Intradimensional shifts tax the ability to attend selectively to the domain of stimulus features that remain the best predictors of biologically significant events. An extradimensional shift occurs when contingencies are reversed such that a previously irrelevant dimension is now rewarded, requiring an attention shift away from the previously relevant dimension. Previous research has shown that medial prefron- tal cortex lesions in rats and monkeys impair extradimensional shifts, but spare intradimensional shifts (Dias et al., 1996; Birrell and Brown, 2000). The present results provide a striking dissociation with the medial prefrontal cortex, revealing a distinct role for the anterior thalamic nuclei within thalamocortical networks that support attentional processing.

Consistent with the initial formation of an intradimensional attentional set, the control animals required decreasing numbers of trials over successive discriminations until forced to shift attention to the previously irrelevant dimension (Chase et al., 2012; Lindgren et al., 2013). In contrast, the anterior thalamic lesions (ATNx1) impaired the acquisition of the intradimensional shift stage. However, when required to attend to the previously irrelevant stimulus dimension (extradimensional shift), the ATNx1 animals did not show an attenuation in performance. Indeed, rather than causing a shift cost, anterior thalamic damage appeared to confer a selective advantage, as the ATNx1 animals acquired the extradimensional shift more rapidly than the previous intradimensional shift. Thus, anterior thalamic damage exerted seemingly opposing effects on set shifting, disrupting intradimensional shifts while at the same time enhancing extradimensional shift performance. As this pattern of results had not previously been reported in rats with neural interventions, the experiment was repeated (ATNx2). Both cohorts showed the same striking pattern of performance.

One seemingly plausible explanation is that anterior thalamic damage disrupts attentional set formation. Impaired attentional set formation would retard the acquisition of intradimensional shifts, as was seen. Interestingly, damage to either the anterior cingulate or posterior cingulate cortices, two sites richly interconnected with the anterior thalamus (Shibata, 1993; Shibata and Naito, 2005; Wright et al., 2010), also disrupts intradimensional set shifting (Ng et al., 2007). However, if the current deficit arose solely because the lesion group attended to both dimensions equally and failed to form an attentional set during the intradimensional shift stage of the procedure, then the extradimensional shift should have been acquired at the same rate as the intradimensional shifts. Remarkably, this was not the case: The anterior thalamic lesion groups acquired the extradimensional shift more rapidly than the previous intradimensional shifts (Fig. 5).

This facilitation of performance on the extradimensional stage might imply that, rather than failing to attend to the relevant perceptual domain (i.e., form an attentional set), anterior thalamic lesion animals attend to the irrelevant perceptual domain (Pearce and Hall, 1980). Attending to irrelevant perceptual dimensions would not only disrupt acquisition of an intradimensional shift but would also facilitate acquisition of the discrimination when that irrelevant dimension becomes relevant during the extradimensional stage. In practice, as animals receive compounds consisting of one stimulus from each perceptual domain, the irrelevant domain remains partially reinforced (Table 1). Over successive intradimensional shifts, normal animals learn to ignore the partially reinforced dimension and instead attend to the dimension that is the most reliable predictor of reinforcement (Mackintosh, 1975). The implication is that damage to the anterior thalamus disrupts the process whereby attention to a stimulus increases if it is the best predictor of reinforcement and, as a corollary, decreases to poor predictors of reinforcement.

To test this hypothesis, we challenged the second cohort of animals with an additional set of discriminations (Table 1). Rats were required to learn about a stimulus dimension (spatial location of the digging pot) that had a history of partial reinforcement over the preceding eight discriminations, as well as a novel stimulus dimension (pot encasing) that did not. As expected, control 
animals took longer to learn that the spatial position of the pot (left or right chamber) now predicted reinforcement. In contrast, anterior thalamic damage again facilitated this extradimensional shift, acquiring this discrimination significantly faster than the sham group (Fig. 6). However, when subsequently required to learn about a novel stimulus dimension that did not have a history of partial reinforcement, no such facilitation effect was seen in the anterior thalamic group, and, if anything, rats in the lesion group were slower to acquire the novel discrimination. These additional discriminations indicate that the critical determinant of whether anterior thalamic lesions facilitate the acquisition of an extradimensional shift is the degree to which the stimulus dimension has been established as an unreliable predictor of reinforcement over successive trials. Equally striking was that this facilitation of extradimensional set shifting occurred when the stimulus dimension involved the spatial properties of the testing environment, even though damage to the rodent anterior thalamus consistently impairs spatial learning (Aggleton and Nelson, 2015). Indeed, this same group of animals (ATNx2 group) was subsequently found to be impaired on a spatial task (Dumont et al., 2014).

One model that anticipates the present results assumes that animals possess two separate attentional mechanisms that are governed by opposing principles, but interact to determine the overall level of attention paid to a stimulus (Pearce and Mackintosh, 2010). According to this proposal, one mechanism increases attention to the stimulus that is the most accurate predictor of a trial outcome, while reducing attention to all other stimuli (Mackintosh, 1975). The second attentional mechanism is also influenced by the degree to which a stimulus, by itself, is an accurate predictor of the outcome. In contrast to the first mechanism, if the stimulus is an accurate predictor of the outcome, then it will receive little attention, but if it is an inaccurate predictor of outcome, then it will receive considerable attention (Pearce and Hall, 1980). This second mechanism ensures that animals devote their associative learning processes maximally to stimuli of uncertain, rather than certain, significance (Pearce and Hall, 1980). The products of both mechanisms are required to explain how reinforcement contingencies affect attention (Pearce and Mackintosh, 2010).

The intriguing implication is that the anterior thalamus is important for the normal operation of the mechanism that directs attention to stimuli that are the best available predictors of the trial outcome. In short, lesions of this region appear to disable this mechanism and leave the Pearce and Hall (1980) uncertainty-based mechanism unimpeded as the sole determinant of the overall level of attention paid to a stimulus. As a consequence, unreliable predictors will command greater attention than reliable signals of reward. Exposure to a new discrimination involving an intradimensional shift will result in attention being directed more to the irrelevant, than the relevant, stimuli and learning will progress slowly. In contrast, if the new discrimination involves an extradimensional shift, then attention will remain on the stimuli that are relevant for the first time, so facilitating discrimination learning.

The anterior thalamic nuclei are well placed to influence behavioral flexibility, given their interconnectivity with prefrontal cortex, anterior cingulate cortex, retrosplenial cortex, and hippocampus. It is, therefore, especially informative that anterior thalamic damage does not reproduce the effects of medial prefrontal lesions, which selectively impair extradimensional set shifting (Birrell and Brown, 2000; Ng et al., 2007), indicating that the medial prefrontal cortex promotes behavioral flexibility by shifting attentional resources away from previously relevant stimuli when reward contingencies change (Sharpe and Killcross, 2014). Furthermore, both prelimbic and mediodorsal thalamic lesions produce perseverative errors when animals are required to shift response rules (Hunt and Aggleton, 1998; Chudasama et al., 2001; Block et al., 2007). Other evidence linking prelimbic cortex with the mediodorsal thalamus for attentional processes comes from lesion studies using the five-choice box where, in contrast, anterior thalamic lesions have no apparent effect (Chudasama and Muir, 2001). This evidence points to a functional dissociation within thalamocortical circuits: the medial prefrontal cortex and mediodorsal thalamus may interact to support disengagement from previously relevant response biases when contingencies change, while the anterior thalamus, perhaps in conjunction with anterior cingulate cortex, regulates attention to task-relevant stimuli. The anterior cingulate cortex is a likely candidate as it is implicated in attentional processes that support reward-guided learning, such as mediating response conflict (MacDonald et al., 2000; Rushworth et al., 2004), error detection (Amiez et al., 2005; Rushworth and Behrens, 2008; Totah et al., 2009), signaling prediction errors, and increasing attention when errors occur (Bryden et al., 2011; Hayden et al., 2011). Perhaps the classic example of these effects is the importance of the anterior cingulate for resolving response conflict on incongruent trials in the Stroop task, when irrelevant information impedes task performance (Pardo et al., 1990). Future experiments will be required to test the specific hypothesis that anterior thalamusanterior cingulate interactions modulate attention to relevant task information. More broadly, the current findings are consistent with other data showing that damage to thalamic nuclei often exerts seemingly different effects on behavior relative to the cortical areas to which they project (Mitchell et al., 2014). The precise mechanism by which these effects occur is currently unclear, but one possibility is that the anterior thalamus modulates information flow between the hippocampus and medial prefrontal cortex through the synchronization of theta (Ketz et al., 2015).

The current results reveal a hitherto unappreciated role for the rodent anterior thalamic nuclei in attentional control. In a striking dissociation from the roles of medial prefrontal cortex and the mediodorsal thalamus, the current study suggests that the anterior thalamic nuclei direct attention to task-relevant stimuli, driving intradimensional set learning at the expense of extradimensional shifts. These results open up new avenues of research into how anterior thalamic nuclei contribute to nonspatial processes (Wolff et al., 2006), as well as providing insights into the neural systems that mediate attention to environmental stimuli that are reliable predictors of biologically significant events. As increased attention to irrelevant or nonpredictive cues has been related to the positive symptoms of schizophrenia (Morris et al., 2013), the current findings may have implications for understanding the neurobiological basis of this disorder (Young et al., 2000).

\section{References}

Aggleton JP, Nelson AJD (2015) Why do lesions in the rodent anterior thalamic nuclei cause such severe spatial deficits? Neurosci Biobehav Rev. Advance online publication. Retrieved February 21, 2015. doi: 10.1016/ j.neubiorev.2014.08.013. CrossRef Medline

Amiez C, Joseph JP, Procyk E (2005) Anterior cingulate error-related activity is modulated by predicted reward. Eur J Neurosci 21:3447-3452. CrossRef Medline

Biesbroek JM, Kuijf HJ, van der Graaf Y, Vincken KL, Postma A, Mali WP, Biessels GJ, Geerlings MI (2013) Association between subcortical vascular lesion location and cognition: a voxel-based and tract-based lesionsymptom mapping study. The SMART-MR study. PLoS One 8:e60541. CrossRef Medline

Birrell JM, Brown VJ (2000) Medial frontal cortex mediates perceptual attentional set shifting in the rat. J Neurosci 20:4320-4324. Medline

Block AE, Dhanji H, Thompson-Tardif SF, Floresco SB (2007) Thalamic- 
prefrontal cortical-ventral striatal circuitry mediates dissociable components of strategy set shifting. Cereb Cortex 17:1625-1636. CrossRef Medline

Bryden DW, Johnson EE, Tobia SC, Kashtelyan V, Roesch MR (2011) Attention for learning signals in anterior cingulate cortex. J Neurosci 31: 18266-18274. CrossRef Medline

Chase EA, Tait DS, Brown VJ (2012) Leisons of the orbital prefrontal cortex impair the formation of attentional set in rats. Eur J Neurosci 36:23682375. CrossRef Medline

Chudasama Y, Muir JL (2001) Visual attention in the rat: a role for the prelimbic cortex and thalamic nuclei? Behav Neurosci 115:417-428. CrossRef Medline

Chudasama Y, Bussey TJ, Muir JL (2001) Effects of selective thalamic and prelimbic cortex lesions on two types of visual discrimination and reversal learning. Eur J Neurosci 14:1009-1020. CrossRef Medline

Dias R, Robbins TW, Roberts AC (1996) Dissociation in prefrontal cortex of affective and attentional shifts. Nature 380:69-72. CrossRef Medline

Duering M, Zieren N, Hervé D, Jouvent E, Reyes S, Peters N, Pachai C, Opherk C, Chabriat H, Dichgans M (2011) Strategic role frontal white matter tracts in vascular cognitive impairment: a voxel-based lesionsymptom mapping study in CADASIL. Brain 134:2366-2375. CrossRef Medline

Dumont JR, Wright NF, Pearce JM, Aggleton JP (2014) The impact of anterior thalamic lesions on active and passive spatial learning in stimulus controlled environments: geometric cues and pattern arrangement. Behav Neurosci 128:161-177. CrossRef Medline

Fox MT, Barense MD, Baxter MG (2003) Perceptual attentional set-shifting is impaired in rats with neurotoxic lesions of posterior parietal cortex. J Neurosci 23:676-681. Medline

Ghika-Schmid F, Bogousslavsky J (2000) The acute behavioral syndrome of anterior thalamic infarction: a prospective study of 12 cases. Ann Neurol 48:220-227. Medline

Hayden BY, Heilbronner SR, Pearson JM, Platt ML (2011) Surprise signals in anterior cingulate cortex: neuronal encoding of unsigned reward prediction errors driving adjustment in behavior. J Neurosci 31:4178-4187. CrossRef Medline

Hunt PR, Aggleton JP (1998) An examination of the spatial working memory deficit following neurotoxic medial dorsal thalamic lesions in rats. Behav Brain Res 97:129-141. Medline

Ketz NA, Jensen O, O’Reilly RC (2015) Thalamic pathways underlying prefrontal cortex-medial temporal lobe oscillatory interactions. Trends Neurosci 38:3-12. CrossRef Medline

Lanna ME, Alves CE, Sudo FK, Alves G, Valente L, Moreira DM, Cavalcanti JLS, Engelhardt E (2012) Cognitive disconnective syndrome by single strategic strokes in vascular dementia. J Neurol Sci 322:176-183. CrossRef Medline

Lindgren HS, Wickens R, Tait DS, Brown VJ, Dunnett SB (2013) Lesions of the dorsomedial striatum impair formation of attentional set in rats. Neuropharmacology 71:148-153. CrossRef Medline

Lombardi WJ, Andreason PJ, Sirocco KY, Rio DE, Gross RE, Umhau JC, Hommer DW (1999) Wisconsin card sorting test performance following head injury: dorsolateral fronto-striatal circuit activity predicts perseveration. J Clin Exp Neuropsychol 21:2-16. CrossRef Medline

MacDonald AW 3rd, Cohen JD, Stenger VA, Carter CS (2000) Dissociating the role of the dorsolateral prefrontal and anterior cingulate cortex in cognitive control. Science 288:1835-1838. CrossRef Medline

Mackintosh NJ (1975) A theory of attention: variations in the associability of stimuli with reinforcement. Psychol Rev 82:276-298. CrossRef

Mamah D, Conturo TE, Harms MP, Akbudak E, Wang L, McMichael AR, Gado MH, Barch DM, Csernansky JG (2010) Anterior thalamic radia- tion integrity in schizophrenia: a diffusion-tensor imaging study. Psychiatry Res 183:144-150. CrossRef Medline

Mitchell AS, Sherman SM, Sommer MA, Mair RG, Vertes RP, Chudasama Y (2014) Advances in understanding mechanisms of thalamic relays in cognition and behavior. J Neurosci 34:15340-15346. CrossRef Medline

Morris R, Griffiths O, Le Pelley ME, Weickert TW (2013) Attention to irrelevant cues is related to positive symptoms in schizophrenia. Schizophr Bull 39:575-582. CrossRef Medline

Nelson AJ, Hindley EL, Haddon JE, Vann SD, Aggleton JP (2014) A novel role for the rat retrosplenial cortex in cognitive control. Learn Mem 21: 90-97. CrossRef Medline

Ng CW, Noblejas MI, Rodefer JS, Smith CB, Poremba A (2007) Double dissociation of attentional resources: prefrontal versus cingulate cortices. J Neurosci 27:12123-12131. CrossRef Medline

Pardo JV, Pardo PJ, Janer KW, Raichle ME (1990) The anterior cingulate cortex mediates processing selection in the Stroop attentional conflict paradigm. Proc Natl Acad Sci U S A 87:256-259. CrossRef Medline

Paxinos G, Watson C (2005) The rat brain in stereotaxic coordinates, Ed 5. New York, NY: Academic.

Pearce JM, Hall G (1980) A modal for Pavlovian learning: variations in the effectiveness of conditioned but not of unconditioned stimuli. Psychol Rev 87:532-552. CrossRef Medline

Pearce J.M, Mackintosh NJ (2010) Two theories of attention: a review and a possible integration. In: Attention and associative learning: from brain to behavior (Mitchell C, Le Pelley M. eds), pp 11-39. Oxford: Oxford UP.

Rushworth MF, Behrens TE (2008) Choice, uncertainty and value in prefrontal and cingulate cortex. Nat Neurosci 11:389-397. CrossRef Medline

Rushworth MFS, Walton ME, Kennerley SW, Bannerman DM (2004) Action sets and decisions in the medial frontal cortex. Trends Cogn Sci 8:410-417. CrossRef Medline

Sharpe MJ, Killcross S (2014) The prelimbic cortex contributes to the down regulation of attention toward redundant cues. Cereb Cortex 24:10661074. CrossRef Medline

Shibata H (1993) Efferent projections from the anterior thalamic nuclei to the cingulate cortex in the rat. J Comp Neurol 330:533-542. CrossRef Medline

Shibata H, Naito J (2005) Organization of anterior cingulate and frontal cortical projections to the anterior and laterodorsal thalamic nuclei in the rat. Brain Res 1059:93-103. CrossRef Medline

Totah NK, Kim YB, Homayoun H, Moghaddam B (2009) Anterior cingulate neurons represent errors and preparatory attention within the same behavioral sequence. J Neurosci 29:6418-6426. CrossRef Medline

Van der Werf YD, Scheltens P, Lindeboom J, Witter MP, Uylings HB, Jolles J (2003) Deficits of memory, executive functioning and attention following infarction in the thalamus; a study of 22 cases with localised lesions. Neuropsychologia 41:1330-1344. CrossRef Medline

Wolff M, Gibb SJ, Dalrymple-Alford JC (2006) Beyond spatial memory: the anterior thalamus and memory for the temporal order of a sequence of odor cues. J Neurosci 26:2907-2913. CrossRef Medline

Wright NF, Erichsen JT, Vann SD, O'Mara SM, Aggleton JP (2010) Parallel but separate inputs from limbic cortices to the mammillary bodies and anterior thalamic nuclei in the rat. J Comp Neurol 518:2334-2354. CrossRef Medline

Wright NF, Vann SD, Erichsen JT, O’Mara SM, Aggleton JP (2013) Segregation of parallel inputs to the anteromedial and anteroventral thalamic nuclei of the rat. J Comp Neurol 521:2966-2986. CrossRef Medline

Young KA, Manaye KF, Liang C, Hicks PB, German DC (2000) Reduced number of mediodorsal and anterior thalamic neurons in schizophrenia. Biol Psychiatry 47:944-953. CrossRef Medline 